\title{
Unraveling the interplay of two counter-rotating helical vortices
}

\author{
Alessandro Capone $\left[\right.$ and Francisco Alves Pereira ${ }^{*}$ * \\ CNR-INM Institute of Marine Engineering, Via di Vallerano 139, 00128 Rome, Italy
}

(Received 7 August 2020; published 12 November 2020)

\begin{abstract}
This paper is associated with a video winner of a 2019 American Physical Society's Division of Fluid Dynamics (DFD) Gallery of Fluid Motion Award for work presented at the DFD Gallery of Fluid Motion. The original video is available online at the Gallery of Fluid Motion, https://doi.org/10.1103/APS.DFD.2019.GFM.V0067.
\end{abstract}

DOI: 10.1103/PhysRevFluids.5.110509

Helical vortices are found in engineering applications when lifting, thrust-, and torque-generating surfaces are rotating, with examples ranging from helicopter rotors to wind turbine to marine propellers. A central aspect of helical vortex systems is in regard to the destabilization processes that trigger their transition from a coherent state in the near wake to a turbulent state in the far wake. The theoretical background on the subject is well outlined (see the seminal work [1]), and several instability modes have been identified: long- and short-wave instabilities of the single vortex core $[2,3]$, self-induced motion due to torsion [4,5], and mutual inductance between neighboring vortex filaments [6].

When two helical vortices are generated, such as in a two-blade rotor, the interplay between the two vorticity systems gives rise to complex phenomena of vortex pairing, merging, break-up, and reconnection [3,7]. Several works have tackled the double-helix configuration experimentally $[6,8]$, theoretically $[9,10]$, and through simulations [11], for this configuration is ideally suited for fundamental understanding while being of great practical significance.

Here visualizations are performed to examine the interaction between two coaxially aligned helical vortices produced by two counter-rotating propellers of slightly different diameters operating in a water tunnel. Imaging of the helical structures is made possible by triggering cavitation in the vortex cores. The results show that the two helical systems merge and split in the regions of closest distance and strongest vorticity, specifically in the blade-tip and near-wake regions [Fig. 1(a)]. Eventually these events lead to a reconnection process that bridges the broken parts, resulting in the periodic formation of isolated vortex rings [Fig. 1(b)]. The rings exhibit a six-lobed sawtooth pattern along the azimuthal direction due to the fact that the propellers are three-bladed and are organized in an alternating manner in the streamwise direction, showing evidence of entrainment, stretching, and roll-up caused by the velocity gradients along the radial direction. We conjecture that the formation of such rings is made possible only by the interacting vortex pairs having the same circulation sign, unlike the cases reported in literature. As such, mutual inductance is expected to be enhanced as a result of the stronger velocity gradients between the interacting vortices, while short-wave disturbances are amplified by the higher strains. In support of this scenario, the circulation difference

\footnotetext{
*Corresponding author: francisco.alvespereira@cnr.it
}

Published by the American Physical Society under the terms of the Creative Commons Attribution 4.0 International license. Further distribution of this work must maintain attribution to the author(s) and the published article's title, journal citation, and DOI. 


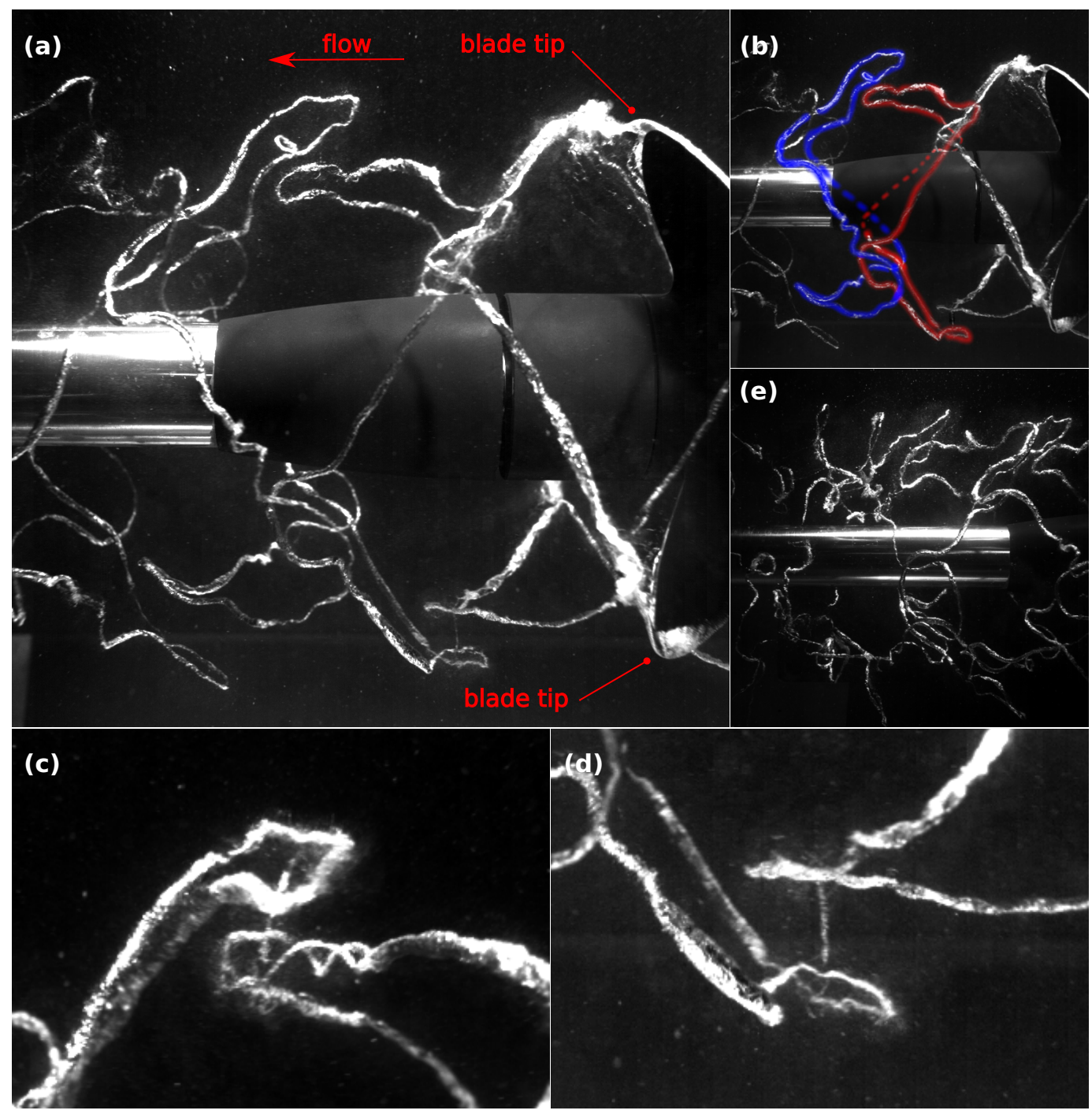

FIG. 1. (a) Two coaxially aligned helical vortices are created by two counter-rotating propellers and interact through merging and break-up processes. (b) Isolated vortex rings arise from reconnection and undergo entrainment, stretching, and roll-up; two consecutive rings are highlighted in red and blue (dashes represent hidden portions of the rings). (c) Circulation gradients between reconnected threads induce twisting and braiding. (d) Intermittent vortical filaments form between consecutive rings. (e) The vortex web maintains high coherence in the near to far wake. The source video is available online at https://doi.org/10.1103/APS. DFD.2019.GFM.V0067.

between reconnecting threads induces torsional effects that affect the local curvature of the vortex filaments, leading to twisted and corkscrew-like patterns [Fig. 1(c)]. Momentum transfer can also be evinced from the formation of vorticity threads that intermittently bridge consecutive rings [Fig. 1(d)]. Finally the helix system remains remarkably coherent as it evolves along the wake, despite the dynamic and continuous rearrangement of the vortical web structure [Fig. 1(e)]. 
The authors wish to thank Dr. Fabio Di Felice, Jais Mohamed, Alessandro Maiocchi, and the CNR-INM technical personnel. F.A.P. also wishes to acknowledge inspiring discussions with Dr. Mohamed Farhat from École Polytechnique Fédérale de Lausanne.

[1] S. E. Widnall, The stability of a helical vortex filament, J. Fluid Mech. 54, 641 (1972).

[2] Y. Hattori and Y. Fukumoto, Short-wave stability of a helical vortex tube: The effect of torsion on the curvature instability, Theor. Comput. Fluid Dyn. 24, 363 (2010).

[3] T. Leweke, S. Le Dizès, and C. H. K. Williamson, Dynamics and instabilities of vortex pairs, Annu. Rev. Fluid Mech. 48, 507 (2016).

[4] R. L. Ricca, The effect of torsion on the motion of a helical vortex filament, J. Fluid Mech. 273, 241 (1994).

[5] J. Boersma and D. H. Wood, On the self-induced motion of a helical vortex, J. Fluid Mech. 384, 263 (1999).

[6] A. Nemes, D. Lo Jacono, H. M. Blackburn, and J. Sheridan, Mutual inductance of two helical vortices, J. Fluid Mech. 774, 298 (2015).

[7] S. Kida and M. Takaoka, Vortex reconnection, Annu. Rev. Fluid Mech. 26, 169 (1994).

[8] H. U. Quaranta, M. Brynjell-Rahkola, T. Leweke, and D. S. Henningson, Local and global pairing instabilities of two interlaced helical vortices, J. Fluid Mech. 863, 927 (2019).

[9] S. C. Crow, Stability theory for a pair of trailing vortices, AIAA J. 8, 2172 (1970).

[10] E. Durán Venegas and S. Le Dizès, Generalized helical vortex pairs, J. Fluid Mech. 865, 523 (2019).

[11] J.-B. Chapelier, B. Wasistho, and C. Scalo, Large-eddy simulation of temporally developing double helical vortices, J. Fluid Mech. 863, 79 (2019). 